Research Paper

\title{
Glucocorticoids improve severe or critical COVID-19 by activating ACE2 and reducing IL-6 levels
}

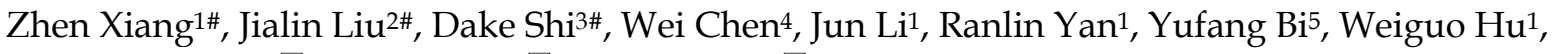 \\ Zhenggang Zhu ${ }^{1 凶}$, Yingyan $\mathrm{Yu}^{1 凶}$, Zhitao Yang ${ }^{6 \bowtie}$ \\ 1. Department of General Surgery of Ruijin Hospital, Shanghai Institute of Digestive Surgery, and Shanghai Key Laboratory for Gastric Neoplasms, Shanghai \\ Jiao Tong University School of Medicine, 200025, Shanghai, China. \\ 2. Department of Critical Care Medicine, Ruijin Hospital, Shanghai Jiaotong University School of Medicine, 200025, Shanghai, China. \\ 3. Department of Infection Control, Ruijin Hospital, Shanghai Jiaotong University School of Medicine, 200025, Shanghai, China. \\ 4. Department of Pulmonary and Critical Care Medicine, Ruijin Hospital, Shanghai Jiaotong University School of Medicine, 200025, Shanghai, China. \\ 5. Department of Endocrinology and Metabolism Disease, Ruijin Hospital, Shanghai Jiaotong University School of Medicine, 200025, Shanghai, China. \\ 6. Emergency Department, Ruijin Hospital, Shanghai Jiaotong University School of Medicine, 200025, Shanghai, China. \\ \#Equal contributions
}

$\triangle$ Corresponding author: Zhitao Yang, email: yangzhitao@hotmail.fr; Tel: 86-21-64370045; Yingyan Yu, email: yingyan3y@sjtu.edu.cn; Tel: 86-21-64370045; Zhenggang Zhu, email: zzg1954@hotmail.com; Tel: 86-21-64370045

() The author(s). This is an open access article distributed under the terms of the Creative Commons Attribution License (https://creativecommons.org/licenses/by/4.0/). See http://ivyspring.com/terms for full terms and conditions.

Received: 2020.05.10; Accepted: 2020.06.17; Published: 2020.06.27

\begin{abstract}
COVID-19 is a public health emergency that has rapidly spread to over 200 countries and regions, and no effective treatment has been established to date. Severe and critical cases have been associated with higher mortality due to acute respiratory distress syndrome (ARDS) and cytokine storm. Based on the novelty and recent emergence of COVID-19, no effective treatment regimen has been identified, thus prompting clinicians to engage in drug repurposing to address the immediate therapeutic need. This study focused on the molecular target angiotensin-converting enzyme 2 (ACE2) of SARS-CoV-2 and screened a group of ACE2 agonists by bioinformatics. Glucocorticoids are a type of ACE2 activator. We verified the efficacy of nine chemicals on regulating ACE2 expression in human GES-1, an upper digestive tract epithelial cell line, and THP-1, a human monocyte cell line, and found that several glucocorticoids imparted activating effects on ACE2 in both cell lines. The drugs triciribine and kinetin riboside activate ACE2 expression or inhibit IL-6 production in macrophages to some extent. In addition, we compared the efficacies of several glucocorticoids. Hydrocortisone showed the strongest effect on ACE2 activation, followed by prednisolone, dexamethasone, and methylprednisolone. We retrospectively analyzed the therapeutic efficacy of nine severe or critical patients from a cohort of 90 COVID-19 cases, who received medium to small doses of glucocorticoids from our integrated medical team in Wuhan. Seven out of nine patients revealed significant improvement in clinical parameters and chest $\mathrm{CT}$ images. This study provides experimental and clinical evidence that medium-to-low-dose glucocorticoids may play a protective role in the respiratory and digestive systems by activating ACE2 and suppressing cytokine storm.
\end{abstract}

Key words: COVID-19; SARS-CoV-2; ACE2; Glucocorticoids; Drug repurposing

\section{Introduction}

From the end of 2019 to the beginning of 2020, an acute respiratory disease known as COVID-19 and caused by SARS-CoV-2 had spread throughout China and the rest of the world. COVID-19, combined with severe acute respiratory syndrome (SARS) in 2002 and Middle East respiratory syndrome (MERS) in 2012, are three zoonotic infectious diseases caused by $\beta$-coronaviruses that occurred in the last 20 years [1-5]. Since $\beta$-coronaviruses are not common pathogens to humans, the general public essentially lacks natural immunity to SARS-CoV-2. Thus, this sudden global pandemic has currently no approved specific antiviral agents, and the development of therapeutic drugs is imperative. 
Similar to SARS-CoV, SARS-CoV-2 uses the receptor, angiotensin-converting enzyme 2 (ACE2), to infect human cells. The virus spike protein (S protein) would bind to the ACE2 of cells, and largely depleting host cells of this specific receptor $[6,7]$. Some researchers have targeted ACE2, blocking the viral S protein from binding to it. ACE2 is a member of the angiotensin-converting enzyme (ACE) family. It was first cloned from $5^{\prime}$ sequencing of a human heart failure ventricle cDNA library in 2000. ACE2 shares homology with ACE in terms of their catalytic domain and provides different key functions in renin-angiotensin-aldosterone system (RAAS). ACE cleaves angiotensin I to generate angiotensin II, whereas ACE2 reduces angiotensin II levels. Thus, ACE2 plays a protective role in the respiratory system, cardiovascular system, and kidneys [8-10]. However, SARS-CoV-2 infection induces a reduction in ACE2 levels and disrupts its protective function in the respiratory and cardiovascular systems, as well as other important organs. Patients diagnosed with COVID-19 rapidly deteriorate to severe or critical conditions $[11,12]$. Therefore, taking inhibitory drugs against SARS-CoV-2 or activating ACE2 may be potential therapeutic strategies for treatment of this infectious disease. Thus, there is an immediate need to identify drugs that could improve multi-organ deterioration in severe or critical COVID-19 patients.

Drug development involves two major strategies. The first strategy consists of screening new drugs from the beginning; however, this is time-consuming and often laborious. The second strategy is drug repurposing, also known as drug repositioning (i.e., older drugs, new uses), which refers to screening drugs with potential intervention of the target. Repurposed drugs requires the approval of the FDA for clinical use or use in clinical trials in order to change its clinical utility $[13,14]$. The latter strategy has the advantages of being fast, safe, and inexpensive. Because old drugs are already on the market, this procedure can save a lot of experiments on drug toxicology, pharmacokinetics, and other pre-experiments. Repurposed drugs are generally safe and have no associated adverse side effects. Drug repurposing research is a big data-driven task that highly relies on bioinformatics and chemical informatics [15,16]. Researchers use computer programs to analyze gene profiles, and then match the small compounds in the database. The candidate compounds are outputted with marked scores. Compounds with higher scores are considered highly reliable. Then, scientists and physicians verify the therapeutic efficacies of candidate compounds by subsequent experiments or clinical practice.

In this study, we used drug repositioning to screen a group of ACE2 agonists (20 compounds) through cross-database analysis. We verified the activating effect of nine available compounds for ACE2 on human epithelial cells and monocytes. Glucocorticoids are candidate agonists of ACE2. We further analyzed our glucocorticoid therapeutic experience involving nine cases of severe and critical COVID-19 from a cohort of 90 patients in Wuhan who were hospitalized by the medical rescue team of Shanghai Ruijin Hospital. Clinically, the safety and effectiveness of glucocorticoids on improving severe and critical COVID-19 have been confirmed.

\section{Results}

\section{The basic expression of ACE2 in 1,019 human tumor cell lines from $\mathbf{2 6}$ tissue types}

We analyzed the ACE2 expression of 1,019 tumor cell lines that originated from 26 types of tissues in the CCLE database. The expression levels and their tissue origin are presented in Figure 1A. Tumor cells from the upper respiratory and digestive tract epithelium exhibited the highest ACE2 expression levels, followed by cell lines of the large intestine, stomach, cervix, and salivary gland. The remaining cell lines from other tissues showed different ACE2 expression levels.

\section{Screening ACE2 agonists by differentially expressed genes between ACE2 ${ }^{\text {high }}$ and ACE2 $2^{\text {low }}$}

Based on mRNA expression levels of ACE2 in 1,019 cell lines, we divided the ACE2 values into high $(n=340)$, medium $(n=339)$, and low $(n=340)$ groups. Three genetic data sets were obtained. We compared the differentially expressed genes between the ACE2 ${ }^{\text {high }}$ and ACE2 ${ }^{\text {low }}$ groups, extracted the top 150 significantly elevated genes (fold-change $\geqslant 1.5, \mathrm{P}<$ 0.01 , Figure 1B), and used these as inputs of the CMap database. The computer matched the compounds that may cause the above differential gene profiles (Figure 1C). The resulting compounds that matched the ACE2 $2^{\text {high }}$ group were considered as ACE2 agonists. Table 1 shows the CMap scores, which represent the matching scores for each compound using 100 full marks, and the closer to 100, the more accurate the match.

\section{ACE2 expression of human epithelial cells could be activated by glucocorticoids or other agonists}

Because the epithelial cells of the respiratory and digestive tracts are susceptible targets of SARS-CoV-2, we verified the regulatory effects of several candidate agonists of ACE2 expression on available normal human epithelial cells. GES-1, a normal digestive 
epithelial cell line, has been maintained in our laboratory. Five commercially available agonists utilized in pandemics, including triciribine, rigosertib, kinetin-riboside, triamcinolone, and cilomilast were assessed. The functions of four commonly used glucocorticoids such as hydrocortisone, prednisolone, methylprednisolone, and dexamethasone were compared. After treatment of GES-1 cells with $100 \mathrm{~nm}$ glucocorticoids or chemicals for $48 \mathrm{~h}$, we examined the protein expression levels of ACE2. Compared to the blank control, hydrocortisone revealed the strongest activating effect on ACE2 expression, followed by prednisolone, dexamethasone, and methylprednisolone. Candidate chemical kinetin riboside also exhibited an activating effect on ACE2 expression (Figure 2A). We further examined the effective doses of hydrocortisone from 0 to $500 \mathrm{~nm}$ and found that the activating effect on ACE2 expression is concentration-dependent, but low doses ranging from 10 to $100 \mathrm{~nm}$ are sufficient in activating ACE2 expression (Figure 2B).

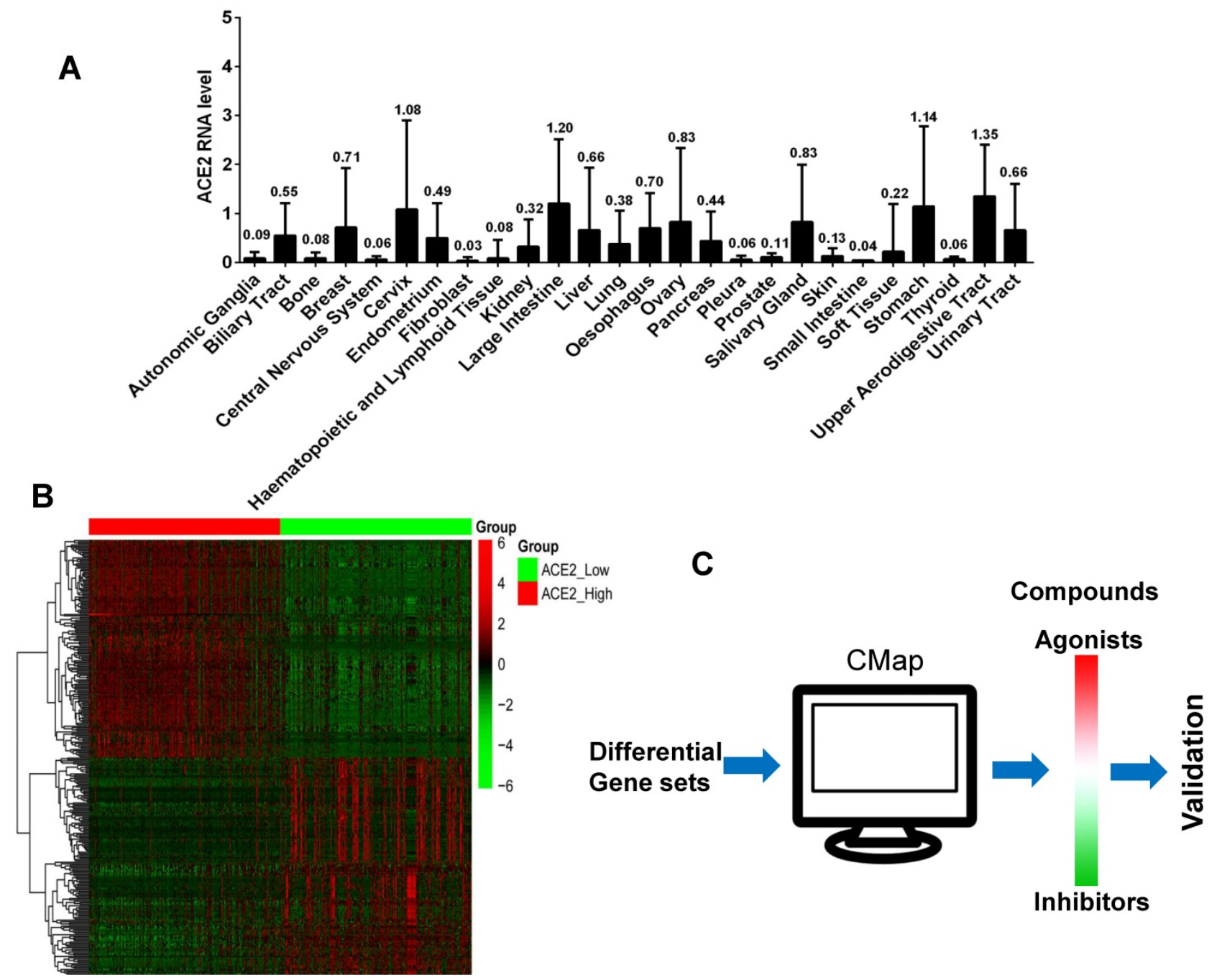

Figure 1. ACE2 expression analysis and candidate agonists screen. A). The mean values of ACE2 gene expression of 1,019 tumor cell lines that originated from 26 types of human tissues. The horizontal axis represents cell line origin, and the vertical axis indicates the mRNA expression level of the ACE2 gene. B). The heat map shows the mRNA expression values (including the ACE2 gene) of 150 highly elevated or significantly decreased differential genes between the ACE2 high and $A C E 2^{\text {low }}$ groups (fold-change $\geq 1.5, P<$ $0.01)$. The red color represents increased mRNA expression levels, and green indicates decreased mRNA expression levels. C). The schematic of agonist screening for ACE2 in the CMap database.

Table 1. The top 20 candidate ACE2 agonists

\begin{tabular}{lll}
\hline Name & Function & CMap score \\
\hline Cytochalasin-d & Actin polymerization inhibitor, actin stabilizer & 98.4 \\
Flubendazole & Microtubule inhibitor, anthelmintic drug & 98.2 \\
Triciribine* & DNA synthesis inhibitor, AKT inhibitor, HIV inhibitor & 97.8 \\
Rigosertib* $^{*}$ & Multi-kinase inhibitor, cell cycle inhibitor, apoptosis inducer & 97.7 \\
Prostratin & NFkB activator, protein kinase activator, HIV inhibitor & 95.2 \\
HG-5-88-01 & Protein kinase inhibitor & 94.7 \\
Quinoclamine & Algicide & 94.4 \\
\hline
\end{tabular}




\begin{tabular}{ll}
\hline HU-211 & Glutamate receptor antagonist, neuroprotective, antioxidant \\
CS-1657 & PARP inhibitor \\
Deforolimus & mTOR inhibitor, angiogenesis inhibitor, immunosuppressant \\
Kinetin-riboside* & Apoptosis inducer, anti-proliferative agent \\
Vinorelbine & Mitosis inhibitor, microtubule inhibitor, apoptosis inducer \\
Triamcinolone* & Corticosteroid hormone receptor agonist, anti-inflammatory agent \\
Perospirone & Dopamine receptor antagonist, antipsychotic agent \\
OMDM-2 & Anandamide uptake inhibitor \\
Eicosatetraynoic-acid & Cyclooxygenase inhibitor, Arachidonic acid uptake inhibitor \\
Cilomilast* & Phosphodiesterase inhibitor, for respiratory disorders (COPD) \\
BRD-K28680267 & Cholecystokinin (CCK) receptor inhibitor \\
Desoxycortone & Mineralocorticoid receptor agonist \\
Phensuximide & Anticonvulsant \\
\hline
\end{tabular}

*Represents the available compounds for subsequent validation experiments
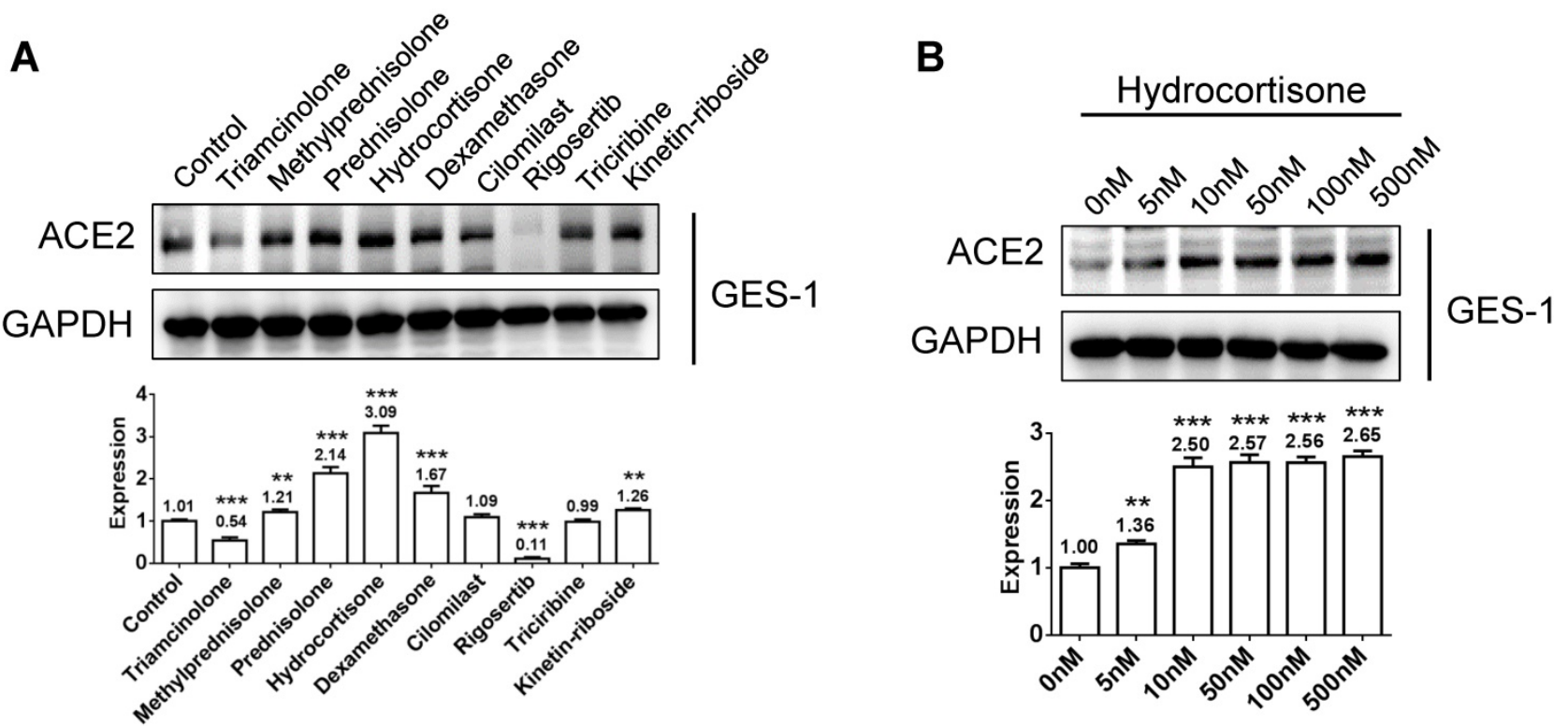

Figure 2. Activating effects of nine chemicals on ACE2 expression in the human gastric epithelial cell GES-1. A) Hydrocortisone revealed the strongest activating effect on ACE2 expression, followed by prednisolone, dexamethasone, and methylprednisolone. The candidate chemical kinetin riboside also exhibited an activating effect on ACE2 expression. B) ACE2 expression induced by hydrocortisone is concentration dependent, although a low dose of 10 to $100 \mathrm{~nm}$ is sufficient to activate ACE2 expression. "*" represents comparison with the "control" or " $0 \mathrm{~nm}$ " groups. $* \mathrm{P}<0.05$, $* * \mathrm{P}<0.01$, $* * * \mathrm{P}<0.001$.

\section{Glucocorticoids impart stronger effects on suppressing interleukin- 6 release and activating ACE2 expression in macrophages compared to other chemicals}

In view of autopsy findings by our medical rescue team in Wuhan that macrophages were aggregated in alveoli and showed elevated ACE2 and cytokine interleukin-6 (IL-6) expression, we induced human monocyte THP-1 cells into M0 (non-activated) and M1 (activated) macrophages, and then tested its activating effect of the above chemicals on ACE2 expression and its inhibitory effect on IL-6 production. After treatment of M0 or M1 macrophages with $100 \mathrm{~nm}$ glucocorticoids or chemicals for $48 \mathrm{~h}$, we examined the protein expression levels of ACE2. Several glucocorticoids and candidate agonists revealed activating ACE2 expression in M0 macrophage, while only glucocorticoids imparted an activating effect on M1 macrophages (Figure 3A). Because hydrocortisone induced the strongest effect on activating ACE2 expression in both M0 and M1 macrophages, we further examined its effective doses using a range from 0 to $500 \mathrm{~nm}$ and found that a low dose of 10 to $100 \mathrm{~nm}$ is sufficient in activating ACE2 expression in macrophages (Figure 3B). Because IL-6 is secreted by M1 macrophages, we incubated M1 macrophages with $100 \mathrm{~nm}$ hydrocortisone for $48 \mathrm{~h}$, and then examined IL-6 content in the supernatant. Compared to the blank control (188.8 pg/mL), all glucocorticoids showed a suppressive effect on IL-6 secretion, although the effect of dexamethasone $(78.2 \mathrm{pg} / \mathrm{mL})$ was the strongest, followed by hydrocortisone (139.9 $\mathrm{pg} / \mathrm{mL})$, methylprednisolone (157.4 pg/mL), triamcinolone $(162.3 \mathrm{pg} / \mathrm{mL})$, and prednisolone $(176$ $\mathrm{pg} / \mathrm{mL}$ ) (Figure $3 \mathrm{C}$ ). The chemical rigosertib also imparted a suppressive effect on IL-6 production. We further examined the effective doses of hydrocortisone on suppressing IL-6 and found that low-dose hydrocortisone within the range of 50 to 100 $\mathrm{nm}$ is sufficient in suppressing IL-6 secretion in M1 macrophages (Figure 3D). 


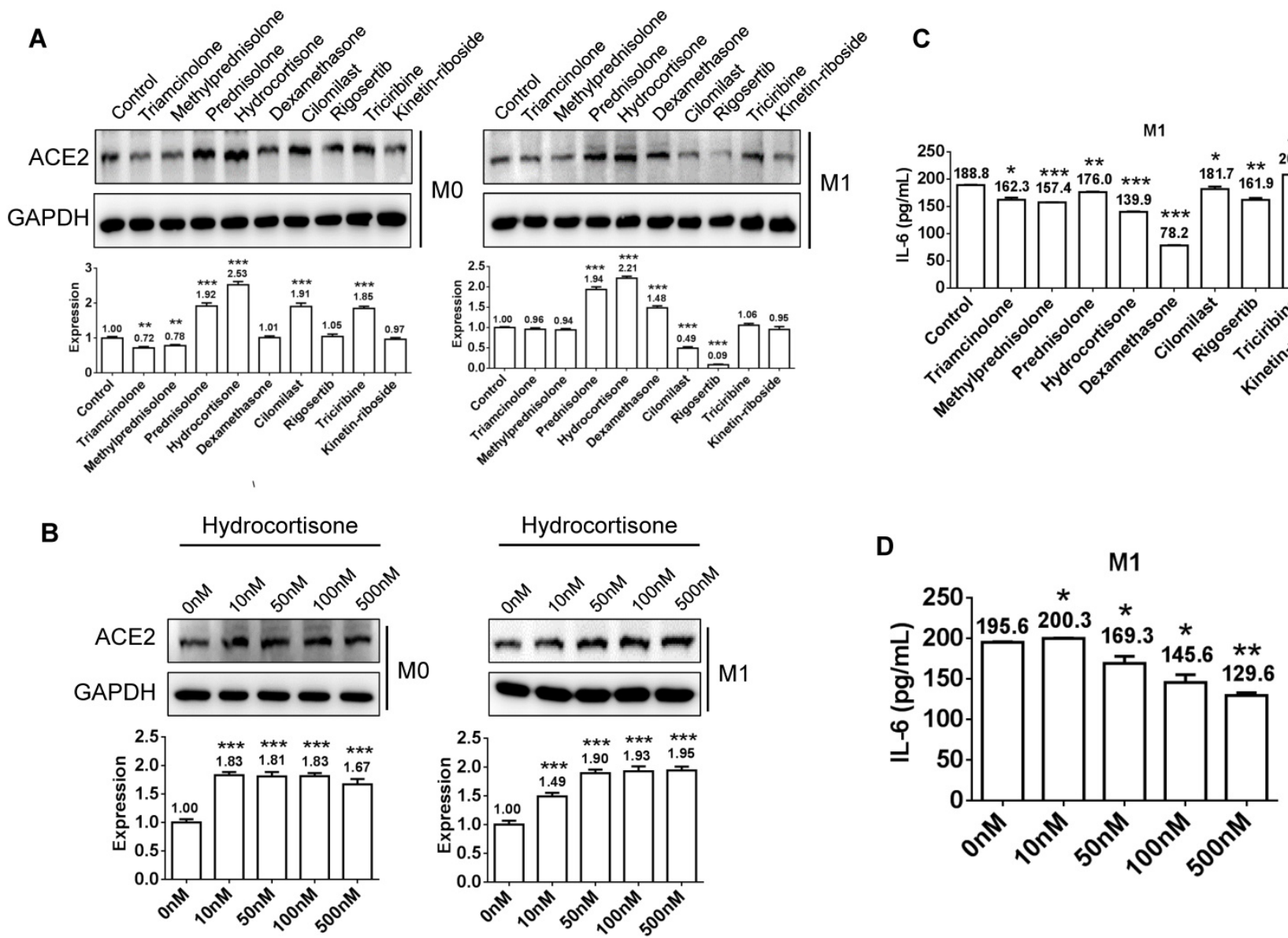

Figure 3. Activation of ACE2 expression and inhibition of IL-6 secretion in macrophages using glucocorticoids or other chemicals. A) Several glucocorticoids and candidate agonists activated ACE2 expression in M0 macrophages, whereas only glucocorticoids imparted an activating effect on M1 macrophages. B) Low-dose hydrocortisone within the range of 10 to $100 \mathrm{~nm}$ is sufficient in activating ACE2 expression in macrophages. C) All glucocorticoids imparted a suppressive effect on IL-6 secretion. Dexamethasone revealed the strongest activating effect, followed by hydrocortisone, methylprednisolone, triamcinolone, and prednisolone. The chemical rigosertib showed a suppressive effect on IL-6 production to some extent. D) Low-dose hydrocortisone within the range of 50 to $100 \mathrm{~nm}$ is sufficient in suppressing IL-6 secretion in MI macrophages. "*” represents a significant difference compared to the "control" or " 0 nm" group. *P $<0.05$, **P $<0.01, * * * \mathrm{P}<0.001$.

Table 2. Therapeutic details of nine severe-to-critical COVID-19 cases using medium-to-low-dose methylprednisolone

\begin{tabular}{|c|c|c|c|c|c|c|c|c|c|c|c|c|c|c|c|}
\hline \multirow[t]{2}{*}{ Case } & \multirow{2}{*}{$\begin{array}{l}\text { Age } \\
\text { /Sex }\end{array}$} & \multirow{2}{*}{$\begin{array}{l}\text { Confirmed } \\
\text { date\$ }\end{array}$} & \multirow{2}{*}{$\begin{array}{l}\text { Oxygen } \\
\text { therapy\# }\end{array}$} & \multirow{2}{*}{$\begin{array}{l}\text { Oxygen } \\
\text { intake\# }\end{array}$} & \multirow{2}{*}{$\begin{array}{l}\text { Disease } \\
\text { type }\end{array}$} & \multicolumn{4}{|c|}{ Vital signs at admission } & \multirow{2}{*}{$\begin{array}{l}\text { Lymphocyte } \\
\text { Before/after* }\end{array}$} & \multirow{2}{*}{$\begin{array}{l}\text { CRP } \\
\text { Before/after* }\end{array}$} & \multirow{2}{*}{$\begin{array}{l}\text { IL-6 } \\
\text { Before/after* }\end{array}$} & \multirow{2}{*}{$\begin{array}{l}\text { Methylpred } \\
\text { treatment } \\
\text { time }\end{array}$} & \multirow{2}{*}{$\begin{array}{l}\text { CT Scan } \\
\text { Before/after* }\end{array}$} & \multirow{2}{*}{ Outcome } \\
\hline & & & & & & HR & $\mathrm{BP}$ & $\mathrm{SaO}_{2}$ & RR & & & & & & \\
\hline 1 & $79 / F$ & 7-day & $\begin{array}{l}\text { Nasal } \\
\text { catheter/ } \\
\text { oxygen mask }\end{array}$ & $3 \mathrm{~L} / \mathrm{min}$ & Critical & 115 & $104 / 77$ & 86 & 35 & $0.58 / 0.67$ & $93.7 / 5.4$ & $35.33 / 5.54$ & 8-day & Yes/Yes & Cured \\
\hline 2 & $76 / F$ & 7-day & $\begin{array}{l}\text { Nasal } \\
\text { catheter/ } \\
\text { oxygen mask }\end{array}$ & $\begin{array}{l}10 \\
\mathrm{~L} / \mathrm{min}\end{array}$ & Critical & 61 & $112 / 54$ & 97 & 20 & $0.43 / 0.48$ & $118.4 / 14.7$ & $5.0 / 5.1$ & 6-day & Yes/Yes & Cured \\
\hline 3 & $45 / F$ & 13-day & Nasal catheter & $5 \mathrm{~L} / \mathrm{min}$ & Severe & 88 & $128 / 81$ & 95 & 20 & $1.20 / 1.24$ & $5 / 0.5$ & ND/ND\& & 6-day & Yes/Yes & Cured \\
\hline 4 & $32 / F$ & 14-day & Nasal catheter & $5 \mathrm{~L} / \mathrm{min}$ & Severe & 78 & $107 / 68$ & 97 & 30 & $2.63 / 2.09$ & $2.6 / 0.5$ & ND/ND\& & 6-day & Yes/Yes & Cured \\
\hline 5 & $44 / \mathrm{M}$ & 2-day & $\begin{array}{l}\text { Nasal } \\
\text { catheter/ } \\
\text { intubation, } \\
\text { ECMO }\end{array}$ & $6 \mathrm{~L} / \mathrm{min}$ & Severe & 78 & $169 / 90$ & 95 & 18 & $0.71 / 1.28$ & $19.5 / \mathrm{ND}$ & $16.98 / 3.01$ & 8-day & Yes/Yes & Cured \\
\hline 6 & $69 / F$ & 12-day & $\begin{array}{l}\text { Oxygen mask/ } \\
\text { intubation }\end{array}$ & $6 \mathrm{~L} / \mathrm{min}$ & Critical & 84 & $195 / 101$ & 93 & 14 & $0.58 / \mathrm{ND}$ & 62.4/ND & $24.26 / \mathrm{ND}$ & 4-day & Yes/No & Death \\
\hline 7 & $68 / F$ & 22-day & $\begin{array}{l}\text { Nasal } \\
\text { catheter/ } \\
\text { intubation }\end{array}$ & $6 \mathrm{~L} / \mathrm{min}$ & Critical & 91 & $130 / 85$ & 84 & 32 & $0.98 / 1.00$ & $62.7 />320$ & $17.48 / 169$ & 7-day & Yes/No & To ICU \\
\hline 8 & $59 / \mathrm{M}$ & 11-day & Nasal catheter & $3 \mathrm{~L} / \mathrm{min}$ & Critical & 122 & $132 / 87$ & 96 & 18 & $0.75 / 1.83$ & $119.1 / 8.2$ & ND/ND\& & 6-day & Yes/Yes & Cured \\
\hline 9 & $57 / \mathrm{M}$ & 16-day & Nasal catheter & $3 \mathrm{~L} / \mathrm{min}$ & Severe & 124 & $141 / 84$ & 91 & 28 & $1.06 / 1.52$ & $68.1 / 4.4$ & $56.4 / 9.94$ & 8-day & Yes/Yes & Cured \\
\hline
\end{tabular}

Note: \$ from appearing symptom to positivity for viral nucleic acid detection. \# represents the status of giving oxygen. *represents count or level before or after methylprednisolone therapy. ND: not done. ND\& means that IL-6 levels were not assessed during the early outbreak stage in the Wuhan hospital. Methylpred: methylprednisolone. $\mathrm{SaO}_{2}$ : arterial oxygen saturation. 


\section{Utilization of glucocorticoids as ACE2 agonist for the treatment of severe or critical COVID-19}

A total of 90 COVID-19 cases were treated by our medical team. Among these, nine severe or critical cases underwent glucocorticoid (methylprednisolone) treatment based on the version 7 guidelines of the National Health Commission of China. Besides oxygen therapy, antiviral therapy, and anti-infection therapy based on the situation, all nine patients were treated with medium-to-low-dose glucocorticoid (methylprednisolone). The medication information is summarized in Figure 4A. The therapeutic dose of methylprednisolone is as follows: $40 \mathrm{mg} / \mathrm{d}$ if body weight $\leqslant 80 \mathrm{~kg}$ for the first 3-4 days, and then 20 $\mathrm{mg} / \mathrm{d}$ for the next 3 days or more with a total of less than 8 days. If body weight is over $80 \mathrm{~kg}, 80 \mathrm{mg} / \mathrm{d}$ for $3-4$ days and $40 \mathrm{mg} / \mathrm{d}$ for the next 3 days or more with a total of less than 8 days (Table 2). We carefully observed the pulmonary CT images and peripheral lymphocyte and serum cytokine levels before or after glucocorticoid treatment. The main parameters assessed in this study included lymphocyte count and C-reactive protein (CRP) and cytokine IL-6 levels (Table 2), which significantly improved with glucocorticoid treatment. Upon radiological examination, we observed various degrees of inflammation absorption in bilateral lungs (Figures 4B and $4 \mathrm{C}$ ) in seven out of nine cases. Two cases were terminated, which consisted of one death due to cytokine storm and another that deteriorated and was transferred to the ICU (Table 2, Cases 6 and 7).

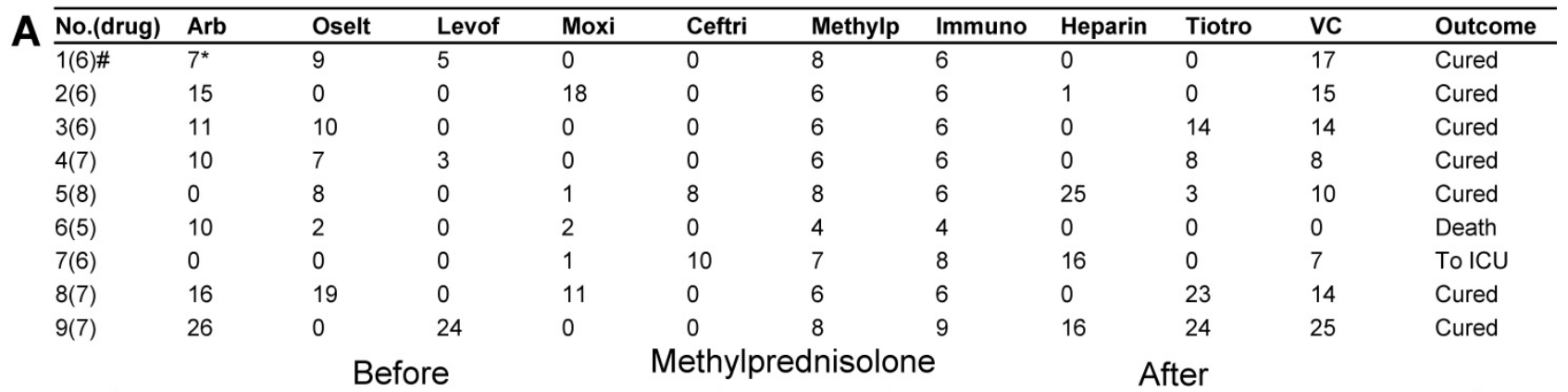

B
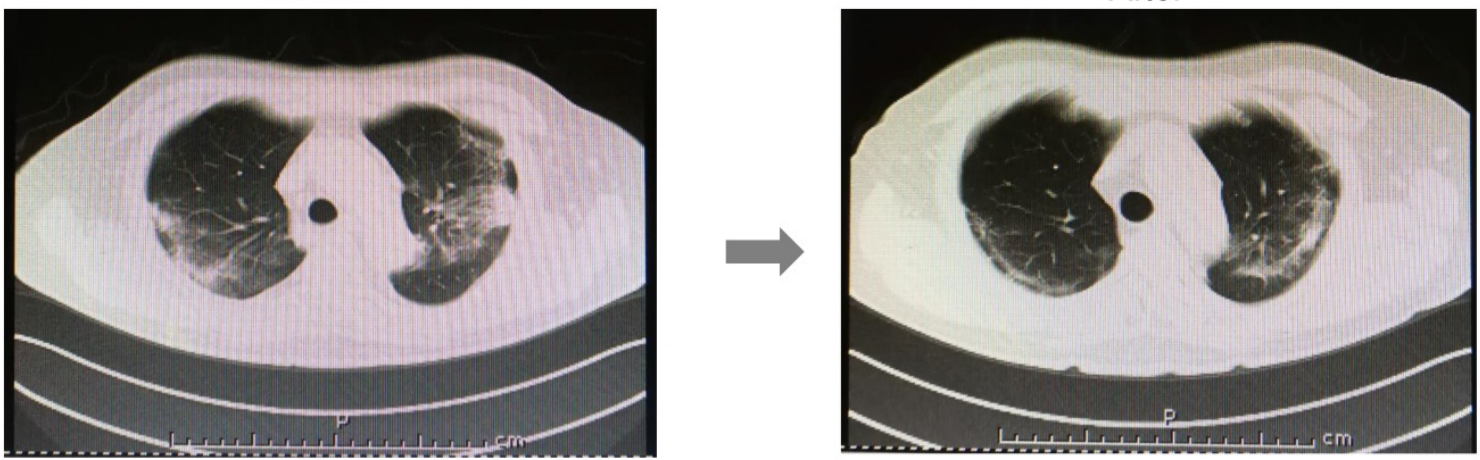

C
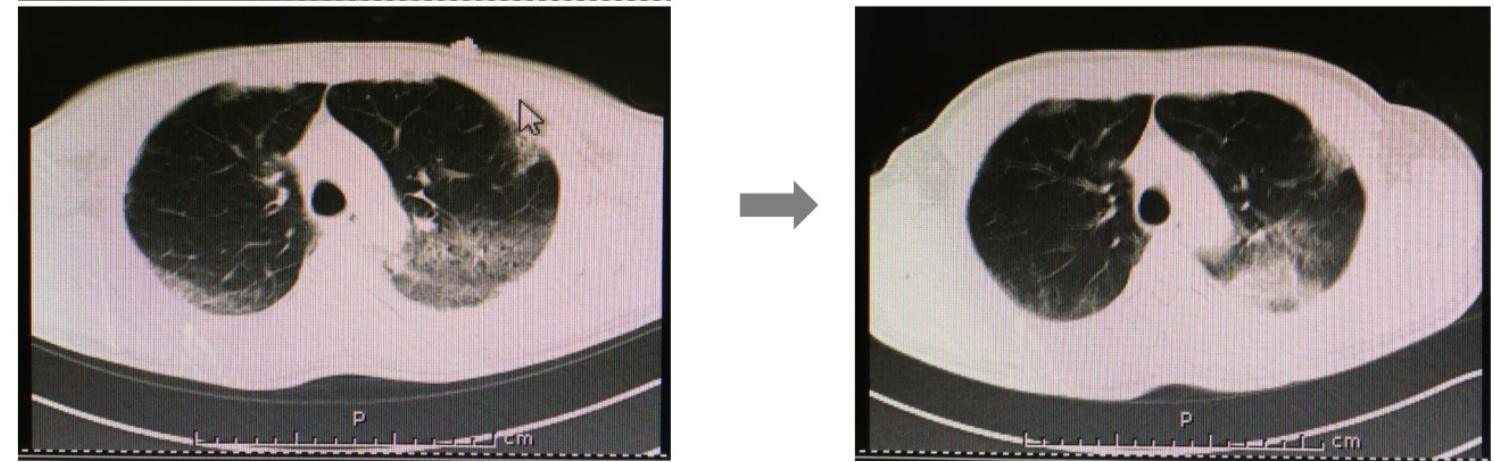

Figure 4. Clinical medication details and improved CT images of nine severe or critical COVID-19 cases. A) The drug names and treatment duration of nine cases. B) Pulmonary CT images before and after methylprednisolone therapy in a case of a 59-year-old man. Left: CT images were obtained before glucocorticoid treatment. Multiple lesions were found in bilateral lungs. Right: CT images obtained after 6-day treatment with glucocorticoids. Absorption of inflammatory lesions in bilateral lungs was observed. C) Pulmonary CT images before and after treatment by methylprednisolone in a case of a 57-year-old man. Left: CT images were obtained before glucocorticoid treatment. Multiple lesions were observed in bilateral lungs. Right: CT images obtained after 8-day methylprednisolone treatment. Inflammation absorption of bilateral lungs to some extent was observed. Drug abbreviations and dosage: Arb: Arbidol (200 mg tid PO); Oselt: Oseltamivir (75 mg bid PO); Levof: Levofloxacin (500 mg/d); Moxi: Moxifloxacin (400 mg/d); Ceftri: Ceftriaxone ( $2 \mathrm{~g} / \mathrm{d})$; Methylp: methylprednisolone; Immuno: Human Immunoglobulin (10 to $15 \mathrm{~g} / \mathrm{d}$ ); Heparin: Low-molecular weight heparin (4,000 IU/d); Tiotro: Tiotropium bromide (400 mg/d PO); VC: Vitamin C (1.5 g/d PO). 


\section{Discussion}

ACE2 is the target of SARS-CoV-2 infection. Several reports have shown that the epithelium of the upper respiratory/digestive tract, gastrointestinal tract, or other tissues expressed higher levels of ACE2 [9-11]. Physiologically, ACE2 plays an important role in maintaining functional balance in vital organs, especially in the respiratory and cardiovascular systems [12,13]. Imai and colleagues found that when ACE2 was knocked out in mice, these would develop acute respiratory distress syndrome (ARDS) due to $S$ protein infection of SARS-CoV [10]. The binding of viral S protein to ACE2 would deplete the host of ACE2, resulting in insufficient protection of the respiratory system, cardiovascular system, and other important organs. Moreover, patients with severe or critical COVID-19 basically receive oxygen therapy. Fang and coworkers reported that when experimental mice were exposed to a hyperoxic environment for 72 $h$, the lungs would exhibit hyperoxic injury accompanied by decreased ACE2 expression. They used an ACE2 agonist to increase ACE2 levels in the lung tissues and inhibited pulmonary inflammation and oxidative stress [17]. Recently, our colleagues reported the results of post-mortem autopsy of two COVID-19 cases in Wuhan, China and found that numerous macrophages had aggregated in the alveoli. These macrophages expressed higher ACE2 and IL-6. The autopsy study suggested that the target cells of SARS-CoV-2 include alveolar macrophages in addition to alveolar epithelium. It has been suggested that cytokine storm is triggered by the release of IL-6 by alveolar macrophages [18]. Khan and coworkers reported the results of an international multi-center clinical trial in ARDS patients with or without using recombinant human ACE2 (rhACE2). All patients who were given rhACE2 showed better tolerance and improvement, including a significant reduction in IL-6 [19]. Because severe or critical COVID-19 patients are subjected to ACE2 depletion, oxygen stress, and cytokine storm, it is essential to deliver ACE2 agonists during therapy.

To rapidly identify potential drugs of ACE2 agonists, we extracted differential gene sets between ACE2 $2^{\text {high }}$ and $A C E 2^{10_{w}}$ cell lines from the public database and used these as inputs into the CMap database for drug repositioning. The CMap database is an extensively used database in drug repositioning research. For instance, Smalley and colleagues used this strategy and identified candidate therapeutic compounds for Huntington's disease, and confirmed that both deferoxamine and chlorzoxazone can improve neurodegeneration lesions by further experimentation [20]. Drug repositioning research relies on newly developed bioinformatics. Accumulating results using human genomics and pharmacogenomics provide us valuable resources for drug screening. For example, the CCLE and CMap databases used in the present study are open databases. The former is a collection of transcriptome information before and after treating 1,019 tumor cell lines from 26 types of human tissues by 24 kinds of compounds [21]. The latter is a database of transcriptome information before and after treating nine human tumor cells by 2,837 compounds [22]. Based on our screening, glucocorticoids were ranked in the top 20 candidate agonists of ACE2.

Glucocorticoids are old anti-inflammatory drugs that have been shown to be very effective in treating asthma and can quickly inhibit the transcription of pro-inflammatory cytokines such as IL-2, IL-3, IL-4, IL-5, and IL-6[23]. Examples of glucocorticoids include prednisolone, triamcinolone, methylprednisolone, and dexamethasone. These drugs are all based on cortisone (hydrocortisone), which has a structural modification that enhances its anti-inflammatory effects. Based on its biological half-life in vivo, glucocorticoids could be further classified based on its length of effect, i.e., short-duration (8 to $12 \mathrm{~h}$ ), medium-duration (12 to 36 h), and long-duration ( 36 to $72 \mathrm{~h}$ ) drugs. For example, cortisone (hydrocortisone) has a biological half-life of 8 to $12 \mathrm{~h}$. Prednisolone, methylprednisolone, or triamcinolone have a biological half-life of 12 to $36 \mathrm{~h}$, whereas that of dexamethasone is 36 to $72 \mathrm{~h}$ [24, 25]. Although triamcinolone, prednisolone, and methylprednisolone are medium-duration drugs, methylprednisolone is preferred for the treatment of lung diseases because it achieves higher concentrations in the lung. Dexamethasone is preferred for the treatment of central nervous system diseases because the penetrating ability into cerebrospinal fluid is superior compared with others [26]. Hydrocortisone is functionally identical to corticoids, but this name is used to distinguish it from endogenous corticoids. As an anti-inflammatory drug, hydrocortisone has been used in the treatment of septic shock at a recommended dose of $200 \mathrm{mg} /$ day and via intravenous administration. Ngaosuwan and colleagues proposed that, in order to reduce steroid-associated complications such as hyperglycemia, a hydrocortisone dose of $100 \mathrm{mg}$ / day might be sufficient. The patients exhibited significantly lower hyperglycemic rates compared with $200 \mathrm{mg} /$ day without increasing mortality in septic shock [27].

Based on the experience during the SARS outbreak in 2002 to COVID-19 outbreak in 2019, the use of glucocorticoids in therapy has remained 
controversial. Shang and colleagues recently reported that low-to-moderate doses of glucocorticoids can be used for the treatment of severe or critical COVID-19, but for not more than 7 days [28]. However, Russell and others expressed a different view, based on the side effects of glucocorticoids during SARS and MERS outbreaks [29]. Recently, the NIH of the USA released treatment guidelines for COVID-19, which proposed that in mechanically ventilated adults with COVID-19 and ARDS, there are insufficient data to recommend either for or against corticosteroid therapy in the absence of another indication, but in COVID-19 patients with refractory shock, low-dose corticosteroid therapy is preferred over no corticosteroid therapy (https://covid19treatment guidelines.nih.gov/). Our study provides convincing evidence that using low-to-medium-dose glucocorticoids for no more than 8 days is safe and beneficial to patients with severe-to-critical COVID-19. However, according to our experiments, hydrocortisone revealed stronger effects on activating ACE2 and inhibiting IL-6 than other glucocorticoids. The discrepancy in the results of our experimental study and bioinformatics prediction may be attributable to differences in cell origins. In bioinformatics prediction, tumor cell lines databases were used, whereas in validating our experiments, immortalized normal cell lines were used. Therefore, the results of any in silico study should be verified by laboratory experiments. Because hydrocortisone resulted in better protective effects on both epithelial cells and macrophages, moderate- to low-dose hydrocortisone (200-100 $\mathrm{mg} /$ day) may be appropriate in treating severe or critical COVID-19 relative to methylprednisolone. However, this needs to be verified in the clinic. In addition, other candidate ACE2 agonists such as kinetin riboside, cilomilast (Ariflo, SB-207499), and rigosertib showed activating effects on ACE2 expression or inhibiting effects on IL-6 production to some extent. However, these were not stronger than glucocorticoids. Whether these could be used in COVID-19 treatment thus requires further investigation.

In conclusion, with the current settings in drug research, the development of new drugs within a short period of time remains a major challenge. Drug repositioning provides a new approach in identifying drugs for specific diseases. The results of this study may be used as a resource in the design of COVID-19 therapeutic agents.

\section{Materials and Methods}

\section{Extraction and analysis of transcriptomic data}

Transcriptomic data of 1,019 tumor cell line from
26 kinds of human tissues was downloaded from the CCLE database (https://portals.broadinstitute. org/ccle). The mRNA expression level of ACE2 was analyzed. The data were divided into three groups based on the expression level of the ACE2 gene, i.e., high expression group $(\mathrm{n}=340)$, medium expression group $(n=339)$, and low expression group $(n=340)$. Then, we further compared differential gene sets between the $\mathrm{ACE} 2^{\text {high }}$ and $\mathrm{ACE} 2^{1{ }^{1} \mathrm{~W}}$ groups and obtained two gene sets from each group. The top 150 genes with the most significant differences in above two gene sets (fold-change $>1.5$-fold, $\mathrm{P}<0.01$ ) were used as input into the Connectivity Map database (CMap, https://clue.io/), and matched corresponding compounds that could cause the above gene profiling changes. The resulting compounds with positive scores were designated as candidate agonists. The full-mark score is 100 . When the score is closer to 100, the candidate compound is more credible.

\section{Cell lines}

Human immortalized gastric epithelium GES-1 and human monocyte THP-1 were cultured in RPMI-1640 containing 10\% FBS and $1 \%$ penicillin-streptomycin and incubated at $37^{\circ} \mathrm{C}$ in a humidified incubator at 5\% $\mathrm{CO}_{2}$. THP-1 cells were induced in RPMI-1640 with $100 \mathrm{ng} / \mathrm{mL}$ phorbol 12-myristate 13-acetate (PMA) for $24 \mathrm{~h}$ for macrophage (M0), and then was further incubated for another $24 \mathrm{~h}$ with $20 \mathrm{ng} / \mathrm{mL}$ IFN-ץ and $10 \mathrm{ng} / \mathrm{mL}$ lipopolysaccharide (LPS) for the activation of macrophages (M1).

\section{Reagents}

Glucocorticoids methylprednisolone and triamcinolone were purchased from MCE (New Jersey, USA). Dexamethasone, hydrocortisone, and prednisolone were purchased from SELLECK (Houston, TX, USA). Chemical triciribine was obtained from SELLECK. Kinetin riboside, cilomilast, rigosertib, PMA, and LPS were purchased from MCE Co. IFN- $\gamma$ was from PeproTech (New Jersey, USA).

\section{Protein expression analysis}

The cell lines were incubated with different chemicals for $48 \mathrm{~h}$, followed by total protein extraction. The protein expression levels of ACE2 (rabbit anti-human ACE2, 1:1,000, AB15348, Cambridge, UK) and HRP-conjugated GAPDH (1:3000, Proteintech, Wuhan, China) were detected by western blotting.

\section{Human IL-6 detection}

The M0 and M1 macrophages were incubated in the above drugs for $48 \mathrm{~h}$, then IL-6 in the supernatants 
was detected by ELISA. A human IL-6 test kit was purchased from Novus (Colorado, USA). Detection was performed according to the product's instructions.

\section{COVID-1 9 patients}

A total of 90 COVID-19 patients were admitted to our medical team. Nine of these cases were evaluated as severe or critical COVID-19 and received glucocorticoid treatment according to the version 7 guidelines of the National Health Commission of China. Severe COVID-19 was diagnosed using the following criteria: $(1)$ respiratory distress ( $\geqslant 30$ breaths/min); (2) oxygen saturation $\leqslant 93 \%$ at rest; (3) arterial partial pressure of oxygen $\left(\mathrm{PaO}_{2}\right) /$ fraction of inspired oxygen $\left(\mathrm{FiO}_{2}\right) \leqslant 300 \mathrm{mmHg}(1 \mathrm{mmHg}=$ $0.133 \mathrm{kPa})$. Cases with chest imaging that showed obvious lesion progression within $24-48 \mathrm{~h}$ of $>50 \%$ were managed as severe cases. Patients meeting any of following conditions were diagnosed as critical COVID-19: (1) respiratory failure and requiring mechanical ventilation; (2) shock; (3) other organ failure that requires ICU care [30]. To evaluate the therapeutic effects, the parameters of lymphocyte ratio and C-reactive protein and IL- 6 cytokine levels were determined before and after glucocorticoid therapy. Chest CT scans of each patient were examined. The clinical study was approved by the Ethics Committee of Ruijin Hospital in Shanghai.

\section{Statistics}

All results of IL- 6 detection were expressed as the mean \pm SD of three repeats. The Student's t-test was conducted using GraphPad Prism 6.0 (CA, USA). Differential gene profiles were plotted using hierarchical clustering by "corrplot" package in $\mathrm{R}$ software. Differences with $\mathrm{P}$ values $<0.05$ were considered significant.

\section{Acknowledgments}

This project was partially supported by the Shanghai Science and Technology Committee (18411953100), the Cross-institute Innovation Foundation of Shanghai Jiao Tong University (YG2017ZD01), the Innovation Foundation of Translational Medicine of Shanghai Jiao Tong University School of Medicine (15ZH4001, TM201617 and TM201702), and the Innovation Foundation for Doctor's Degree (BXJ201914). We acknowledge the open database of CCLE and CMap. We also thank the staff of Ruijin Hospital COVID-19 Critical Care Medical Team. We thank LetPub (www.letpub.com) for its linguistic assistance during the preparation of this manuscript.

\section{Author contributions}

YY Yu, ZT Yang, and Z Xiang were involved in the concept and design of the research study. Z Xiang, $\mathrm{J} \mathrm{Li}$, and RL Yan assisted in the experiments. JL Liu, DK Shi, W Chen, YF Bi, WG Hu, and ZT Yang treated the COVID-19 patients. ZG Zhu and YY Yu discussed and wrote the manuscript.

\section{Competing Interests}

The authors have declared that no competing interest exists.

\section{References}

1. Snijder EJ, van der Meer $Y$, Zevenhoven-Dobbe J, Onderwater JJ, van der Meulen J, Koerten HK, et al. Ultrastructure and Origin of Membrane Vesicles Associated with the Severe Acute Respiratory Syndrome Coronavirus Replication Complex. J Virol. 2006; 80(12):5927-5940.

2. Chan JF, Lau SK, To KK, Cheng VC, Woo PC, Yuen KY. Middle East Respiratory Syndrome Coronavirus: Another Zoonotic Betacoronavirus Causing SARS-Like Disease. Clin Microbiol Rev. 2015; 28(2):465-522.

3. Chan JF, Kok KH, Zhu Z Chu H, To KKW Yuan S, et al Genomic characterization of the 2019 novel human-pathogenic coronavirus isolated from a patient with atypical pneumonia after visiting Wuhan. Emerg Microbe Infect. 2020; 9(1):221-236.

4. Zhu N, Zhang D, Wang W, Li X, Yang B, Song J, et al. A Novel Coronavirus from Patients with Pneumonia in China, 2019. N Engl J Med. 2020; 382(8):727-733.

5. Gralinski LE, Menachery VD. Return of the Coronavirus: 2019-nCoV. Viruses. 2020; 12 (2 pii: E): 135.

6. Cao Y, Li L, Feng Z, Wan S, Huang P, Sun X, et al. Comparative genetic analysis of the novel coronavirus (2019-nCoV/SARS-CoV-2) receptor ACE2 in different populations. Cell Discov. 2020; 6:11.

7. Turner AJ, Hiscox JA, Hooper NM. ACE2: from vasopeptidase to SARS virus receptor. Trends Pharmacol Sci. 2004; 25(6):291-294.

8. Donoghue M, Hsieh F, Baronas E, Godbout K, Gosselin M, Stagliano N, et al A novel angiotensin-converting enzyme-related carboxypeptidase (ACE2) converts angiotensin I to angiotensin 1-9. Circ Res. 2000;87(5):E1-E9.

9. Tipnis SR, Hooper NM, Hyde R, Karran E, Christie G, Turner AJ. A human homolog of angiotensin-converting enzyme. Cloning and functional expression as a captopril-insensitive carboxypeptidase. J Biol Chem. 2000; 275(43):33238-33243.

10. Imai $\mathrm{Y}, \mathrm{Kuba} \mathrm{K}$, Penninger JM. The discovery of angiotensin-converting enzyme 2 and its role in acute lung injury in mice. Exp Physiol. 2008; 93(5):543-8.

11. Zheng J, Li G, Chen S, Bihl J, Buck J, Zhu Y, et al. Activation of the ACE2/Ang-(1-7)/Mas pathway reduces oxygen-glucose deprivation-induced tissue swelling, ROS production, and cell death in mouse brain with angiotensin II overproduction. Neuroscience. 2014; 273:39-51.

12. Delavan B, Roberts R, Huang R, Bao W, Tong W, Liu Z. Computational drug repositioning for rare diseases in the era of precision medicine. Drug Discov Today. 2018; 23:382-394.

13. Langedijk I, Mantel-Teeuwisse AK, Slijkerman DS, Schutjens MH. Drug repositioning and repurposing: terminology and definitions in literature. Drug Discov Today. 2015; 20(8):1027-34.

14. Manzotti G, Parenti S, Ferrari-Amorotti G, Soliera AR, Cattelani S, Montanari $\mathrm{M}$, et al. Monocyte-macrophage differentiation of acute myeloid leukemia cell lines by small molecules identified through interrogation of the Connectivity Map database. Cell Cycle. 2015; 14:2578-89.

15. Arakelyan A, Nersisyan L, Nikoghosyan M, Hakobyan S, Simonyan A, Hopp L, et al. Transcriptome-Guided Drug Repositioning. Pharmaceutics. 2019; 11(12 pii: E): 677. doi: 10.3390/pharmaceutics11120677.

16. Liu W, Tu W, Li L, Liu Y, Wang S, Li L, et al. Revisiting Connectivity Map from a gene co-expression network analysis. Exp Ther Med. 2018; 16(2):493-500.

17. Fang Y, Gao F, Liu Z. Angiotensin-converting enzyme 2 attenuates inflammatory response and oxidative stress in hyperoxic lung injury by regulating NF-KB and Nrf2 pathways. QJM. 2019; 112(12):914-924.

18. Wang CF, Xie J, Zhao L, Fei X, Zhang H, Tan Y, et al. Aveolar macrophage activation and cytokine storm in the pathogenesis of severe COVID-19. Research Square. 2020 March 25;DOI: 10.21203/rs.3.rs-19346/v1

19. Khan A, Benthin C, Zeno B, Albertson TE, Boyd J, Christie JD, et al. A pilot clinical trial of recombinant human angiotensin-converting enzyme 2 in acute respiratory distress syndrome. Crit Care. 2017; 21(1):234.

20. Smalley JL, Breda C, Mason RP, Kooner G, Luthi-Carter R, Gant TW, et al. Connectivity mapping uncovers small molecules that modulate neurodegeneration in Huntington's disease models. J Mol Med (Berl). 2016; 94(2):235-45. 
21. Barretina J, Caponigro G, Stransky N, Venkatesan K, Margolin AA, Kim S, et al. The Cancer Cell Line Encyclopedia enables predictive modelling of anticancer drug sensitivity. Nature. 2012; 483:603-7.

22. Lamb J, Crawford ED, Peck D, Modell JW, Blat IC, Wrobel MJ, et al. The Connectivity Map: using gene-expression signatures to connect small molecules, genes, and disease. Science. 2006; 313:1929-1935.

23. Barnes PJ. Glucocorticosteroids: current and future directions. Br J Pharmacol. 2011; 163(1): 29-43.

24. Adcock IM, Mumby S. Glucocorticoids. Handb Exp Pharmacol. 2017; 237: 171-196.

25. Kapugi M and Cunningham K. Corticosteroid. Orthopaedic Nursing. 2019; 38(5): 336-338.

26. Czock D, Keller F, Rasche FM, Haussler H. Pharmacokinetics and Pharmacodynamics of Systemically Administered Glucocorticoids. Clin Pharmacokinet. 2005; 44 (1): 61-98.

27. Ngaosuwan K, Ounchokdee K, Chalermchai T. Clinical Outcomes of Minimized Hydrocortisone Dosage of $100 \mathrm{Mg}$ /Day on Lower Occurrence of Hyperglycemia in Septic Shock Patients. Shock. 2018; 50(3): 280-285.

28. Shang L, Zhao J, Hu Y, Du R, Cao B. On the use of corticosteroids for 2019-nCoV pneumonia. Lancet; 2020;395(10225):683-684.

29. Russell CD, et al. Clinical evidence does not support corticosteroid treatment for 2019-nCoV lung injury. Lancet. 2020; 395(10225):683-684.

30. National Health Commission \& National Administration of Traditional Chinese Medicine. Diagnosis and treatment protocol for novel coronavirus pneumonia (Trial version 7). Chin Med J (Engl). 2020; 133(9):1087-1095. 